\title{
Case of Corneal Perforation as a Complication after Uneventful CXL without Infection
}

\author{
Goktug Demirci, Akif Ozdamar
}

\section{ABSTRACT}

Purpose: To demonstrate that UVA/riboflavin crosslinking (CXL) can cause corneal melting in some unpredictable cases.

Materials and methods: We will present a case report of 25-year-old male patient referred for emergency keratoplasty which is due to corneal melting and perforation developed after uneventful CXL procedure for the treatment of keratoconus.

Results: We performed uncomplicated penetrating keratoplasty of the left eye in our hospital.

Conclusion: CXL is gradually becoming the standard treatment procedure for progressive keratoconus and the only major safety concerns associated with CXL are ocular surface damage and endothelial cell damage. This case has shown, however, that even if CXL seems to have been successful, there is still a risk of adverse outcomes. Our report suggests that further research is necessary regarding the safety of corneal collagen crosslinking in corneas with keratoconus.

Keywords: Corneal melt, CXL, Enzymatic activity and keratoconus, Penetrating keratoplasty.

How to cite this article: Demirci G, Ozdamar A. Case of Corneal Perforation as a Complication after Uneventful CXL without Infection. J Kerat Ect Cor Dis 2013;2(3):139-142.

\section{Source of support: Nil}

Conflict of interest: None declared

\section{INTRODUCTION}

Keratoconus is a progressive ectasia of the cornea resulting from noninflammatory thinning of the corneal stroma. ${ }^{1}$ Early experiments by Cannon and Foster implicated the role of degraded normal collagen or synthesis of abnormal collagen in the pathogenesis of keratoconus. ${ }^{2}$ Until the mid-1990s, the only treatment for ectasia was penetrating keratoplasty, but in 1997, Spoerl et al showed that corneal rigidity is increased following corneal crosslinking (CXL) treatment with riboflavin and UV-A in vitro experiments. ${ }^{3}$ Thereafter, CXL treatment has become the standard treatment protocol for ectatic disorders, such as keratoconus and pellucid marginal degeneration.

In the literature, although CXL is thought to be a safe procedure, there are reported complications such as sterile infiltrates, stromal scars, delayed epithelial healing, and bacterial keratitis which mostly occurs in the healing period of epithelium. ${ }^{4-6}$

\section{CASE REPORT}

A 25-year-old male patient was admitted to our cornea department for keratoplasty due to the complications of melting and perforation from a previously performed CXL with UV-A for the treatment of keratoconus in the left eye. In his epicrisis, obtained from the referring surgeon, before the CXL procedure his visual acuity was 0.7 OD 0.2 OS. Amblyopia was present in the left eye. He had bilateral keratoconus according to corneal topographies (Figs 1 and 2). The central corneal thickness was 447 microns for both eyes. The thickness of the thinnest corneal point of the left eye was 425 microns. The fundus examination was bilateral normal. He had no known allergy, no immune disease, and no systemic disease. Standard CXL treatment was performed in the patient's left eye. Examination with biomicroscopy showed that he only had epithelial defect of the central cornea on the 5th day. He had a small thinning in the cornea on the 11th day. Despite antibiotic drops and contact lens, the melting process progressed and perforation developed without any sign of infection or reaction. All cultures taken from the cornea were negative. To maintain the anterior chamber, the perforation was sutured (Fig. 3). We performed uncomplicated penetrating keratoplasty of the left eye in our hospital (Fig. 4).

\section{DISCUSSION}

Keratoconus is a bilateral, noninflammatory corneal ectasia with an incidence of approximately one per 2,000 in the general population. ${ }^{7}$ Unlike all previous keratoconus management techniques, the only approach designed to slow, stabilize, or even possibly reverse the progression of corneal ectasia in the early phase of the condition is $\mathrm{CXL}{ }^{8}$

Since Spoerl et al introduced CXL as a treatment method for keratoconus, many studies have been conducted to investigate safety issues related to this technique. ${ }^{8}$ The possible potential damage to ocular tissue during CXL can be direct or indirect. As reported in the current literature, direct complications are stromal haze, which in most cases resolves within 1 year and endothelial cell damage; because the procedure has an effect on both anterior and posterior stroma very near to the endothelial cell layer, the most critical part of the eye to suffer from damage, either directly due 


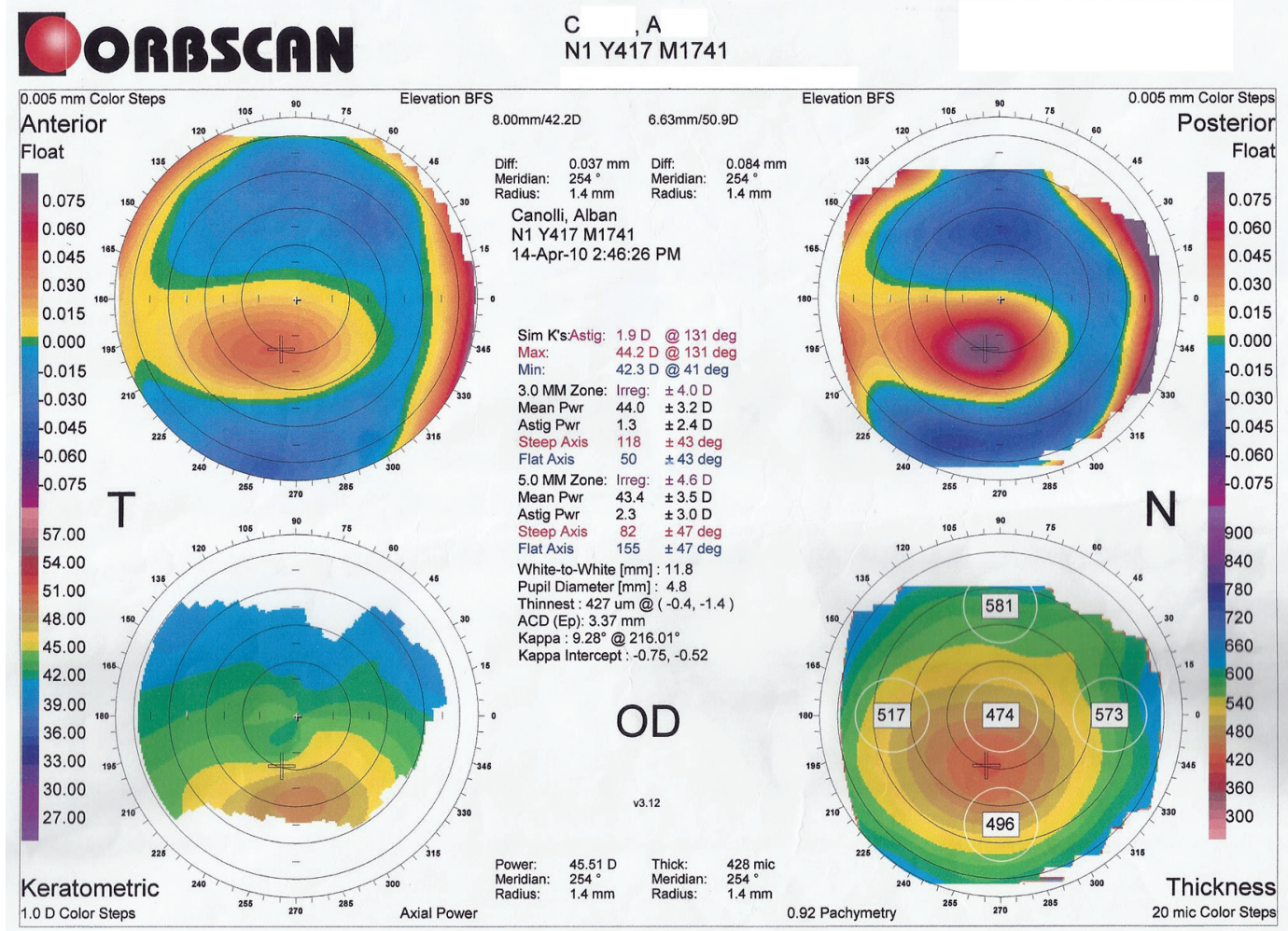

Fig. 1: Corneal topography of the right eye

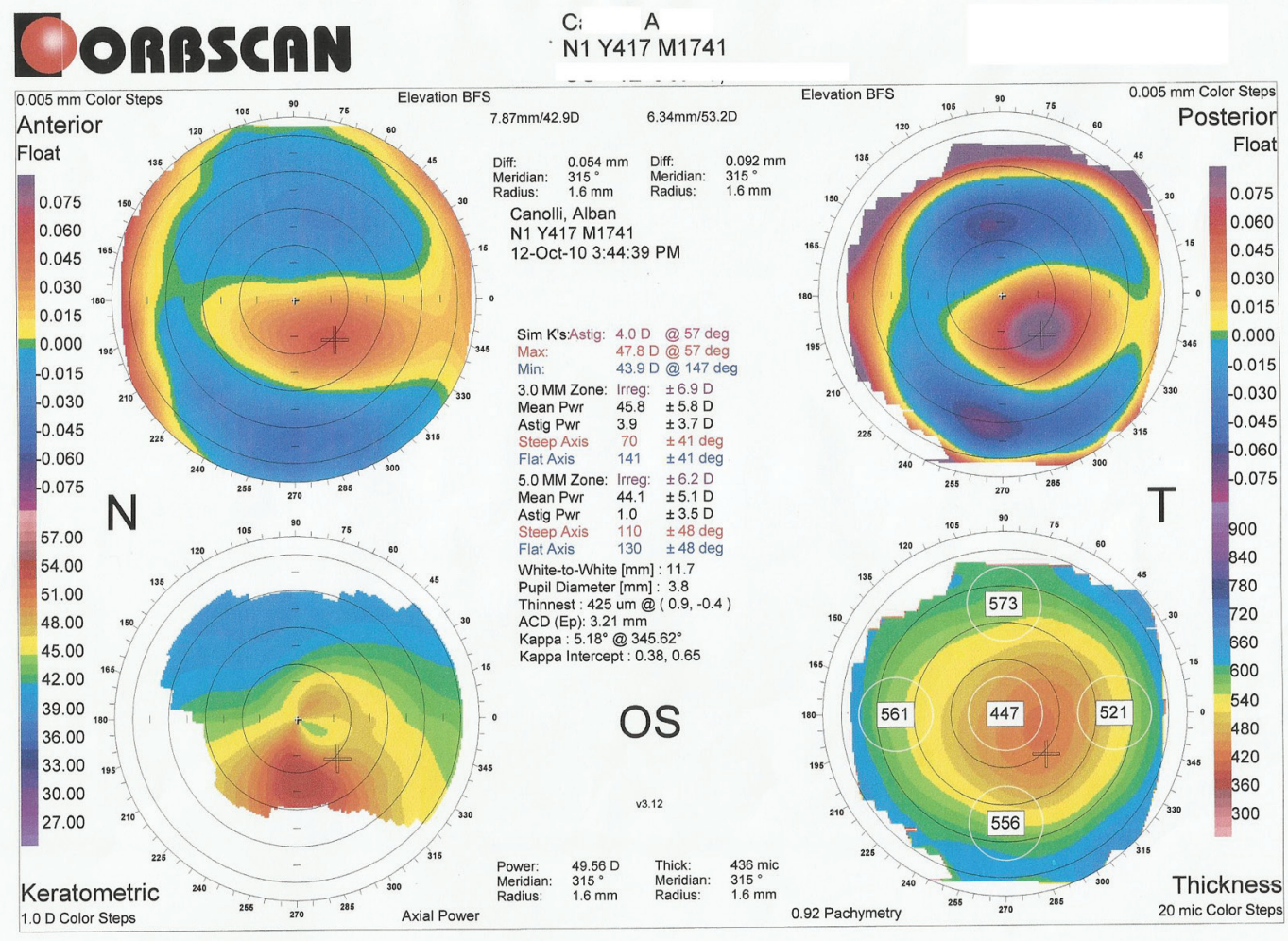

Fig. 2: Corneal topography of the left eye before CXL

to from UV light or due to the free radicals, is the corneal endothelium. ${ }^{8,9}$ Spoerl et al showed that after CXL, the stroma is depopulated of keratocytes to approximately 300 microns deep. Repopulation of this area takes up to 6 months. As long as the treated cornea has a minimum thickness of
400 microns (as recommended), the corneal endothelium will not experience damage, nor will deeper structures such as the lens and retina. ${ }^{8}$ Kymionis et al found that CXL in thin corneas with a minimum thickness of less than $400 \mu \mathrm{m}$ after epithelial removal resulted in a significant decrease in 


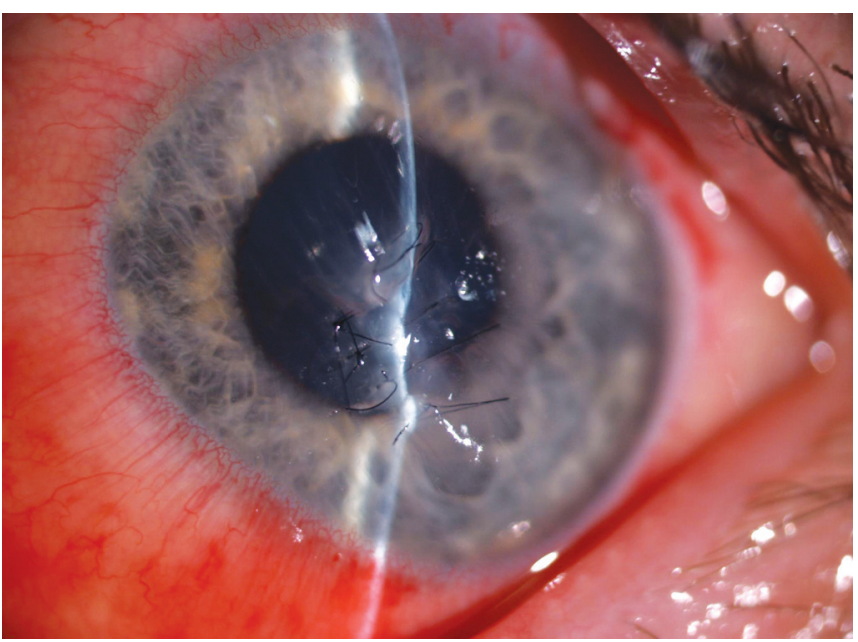

Fig. 3: Sutured corneal perforation of the left eye

endothelial cell density. This finding was not related to other intraoperative or postoperative complications. ${ }^{10}$

Indirect complications are peripheral sterile infiltrates and keratitis, which is mostly acquired after the procedure. ${ }^{11,12}$ This is mostly due to the presence of an epithelial defect, the use of soft bandage contact lenses, and topical corticosteroids used in the immediate postoperative period. Infectious agents likely emerge during the early postoperative period rather than during surgery because CXL not only damages keratocytes, but also kills bacteria and fungi. ${ }^{11-13}$

After an extensive literature search, we found only four cases of corneal melt and perforation occurring after CXL without infection. In the first case, the reason for the side effects was not known, but diclofenac and proparacaine were thought to be responsible for acute inflammation. That case had very significant inflammation, affecting nearly $2 / 3$ of the cornea and it is significantly different from ours because in our case, there was only a small epithelial defect in the apex of the cornea. ${ }^{14}$ The second case in the literature was a Down syndrome patient in which the CCT was below 400 micron, which is not within the safety guidelines. ${ }^{15}$ The third and last case of melt after CXL without infection was reported from Greece. Here, as in the first case, the reason for the side effects could not found, but this also differed from our case in terms of the clinical and biomicroscopic findings. The patient's symptoms were very acute and the epithelial defect was large; moreover, the corneal haze was deep and very large, representing an acute inflammatory response. ${ }^{16}$ Keratitis after CXL procedure is not surprising in a deepithelized cornea after contact with a microorganism. In our case the cornea and anterior chamber were very quiet. There was no keratitis, systemic and local disease found and there was just a small epithelial defect that ended with perforation and loss of the anterior chamber. This was most probably due to increased local keratocyte damage after the procedure; this should be further investigated.

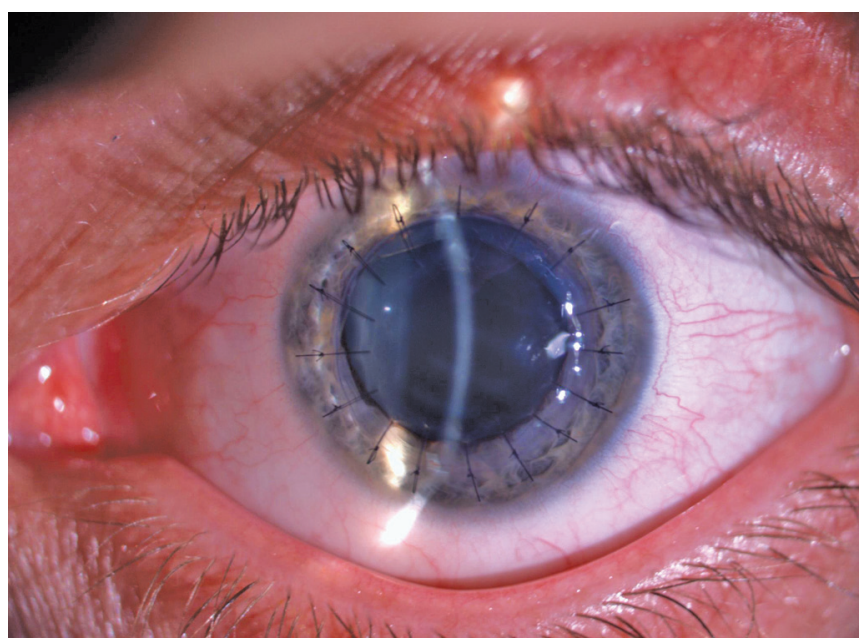

Fig. 4: Penetrating keratoplasty of the left eye

\section{CONCLUSION}

CXL is gradually becoming the standard treatment procedure for progressive keratoconus and the only major safety concerns associated with CXL are ocular surface damage and endothelial cell damage. This case has shown, however, that even if CXL seems to have been successful, there is still a risk of adverse outcomes. Our report suggests that further research is necessary regarding the safety of corneal collagen crosslinking in corneas with keratoconus. CXL should therefore be performed only by surgeons with the ability to perform corneal transplantation, and patients should be prepared for the worst scenario.

\section{REFERENCES}

1. Krachmer JH, Feder RS, Belin MW. Keratoconus and related noninflammatory corneal thinning disorders. Surv Ophthalmol 1984;28:293-322.

2. Cannon DJ, Foster CS. Collagen crosslinking in keratoconus. Invest Ophthalmol Vis Sci 1978;17:63-65.

3. Spoerl E, Huhle M, Kasper M, Seiler T. Increased rigidity of the cornea caused by intrastromal crosslinking. Ophthalmology 1997;94:902-906.

4. Raiskup F, Hoyer A, Spoerl E. Permanent corneal haze after riboflavin-UVA-induced crosslinking in keratoconus. J Refract Surg 2009;25:824-828.

5. Koppen C, Vryghem JC, Gobin L. Keratitis and corneal scarring after UVA/riboflavin crosslinking for keratoconus. J Refract Surg 2009;25:819-823.

6. Pérez-Santonja JJ, Artola A, Javaloy J. Microbial keratitis after corneal collagen crosslinking. J Cataract Refract Surg 2009;35:1138-1140.

7. Rabinowitz YS. Keratoconus. Surv Ophthalmol 1998 Jan-Feb; 42:297-319.

8. Spoerl E, Hoyer A, Pillunat LE, Raiskup F. Corneal crosslinking and safety issues. Open Ophthalmol J 2011;11:14-16.

9. Wollensak G, Spoerl E, Seiler T. Increased resistance of riboflavin/ultraviolet: a treated cornea against enzymatic digestion. Curr Eye Res 2004;29:35-40.

10. Kymionis GD, Grentzelos MA, Kounis GA. Intraocular pressure measurements after corneal collagen crosslinking with riboflavin 
and ultraviolet A in eyes with keratoconus. J Cataract Refract Surg 2010;36:1724-1727.

11. Dhawan S, Rao K, Natrajan S. Complications of corneal collagen crosslinking. J Ophthalmol 2011; Available at: http://www.ncbi. nlm.nih.gov/pmc/articles/PMC3255294/

12. Rama P, Di Matteo F, Matuska S, Paganoni G, Spinelli A. Acanthamoeba keratitis with perforation after corneal crosslinking and bandage contact lens use. J Cataract Refract Surg 2009;35:788-791.

13. Mangioris GF, Papadopoulou DN, Balidis MO. Corneal infiltrates after corneal collagen crosslinking. J Refract Surg 2010;26:609-611.

14. Gokhale NS, Vemuganti GK. Diclofenac-induced acute corneal melt after collagen crosslinking for keratoconus. Cornea 2010;29:117-119.

15. Faschinger C, Kleinert R, Wedrich A. Corneal melting in both eyes after simultaneous corneal crosslinking in a patient with keratoconus and Down syndrome. Ophthalmology 2010;107:951-955.

16. Labiris, et al. Journal of Medical Case Reports 2011; 5:152. Available at: http://www.jmedicalcasereports.com/ content $/ 5 / 1 / 152$.

\section{ABOUT THE AUTHORS}

\section{Goktug Demirci (Corresponding Author)}

Assistant Professor, Department of Ophthalmology, Istanbul Medipol University, Istanbul, Turkey, Phone: 902126311522, e-mail: drgdemirci@yahoo.com

\section{Akif Ozdamar}

Professor, Department of Ophthalmology, Istanbul University Cerrahpasa Medical Faculty, Istanbul, Turkey 